\title{
The Study of Sand-blocking Characteristic and Flow Field Simulation Analysis of the Ring-shaped Sand-barrier
}

\author{
Wei Qiang ZHENG, Wen Jun WEI, Ping Yi LIU * \\ China Agricultural University, Beijing100083, China
}

\begin{abstract}
Aiming at the complexity of wind direction and irregular sand flow in a desert area, a combinatorial ring-shaped sand barrier is used. Stokes law of inertia force and centrifugal force and gravity sedimentation are used. With CFD fluid software Fluent, laminar flow model Equation and $\kappa-\varepsilon$ turbulence model, the wind speed of the sand in the sand-gas-solid two-phase flow passing through the circular sand barrier is studied at different distances and different altitudes after the sand barrier, the wind speeds before and after the sand barrier are compared and analyzed. The mean minimum wind speed behind the single sand barrier was reduced by $32.5 \%-49.4 \%$ compared with that before the sand barrier. The wind speed at different height of the composite sand barrier was reduced by $30 \%-58.3 \%$ compared with the inlet wind speed, which solved the problem of irregular wind and sand control in the desert wind direction.
\end{abstract}

\section{Introduction}

Sand particles forms aeolian flow under the action of wind farms, the aeolian flow aggravates the process of land desertification, study on the law of sand flow, master the law of sand flow, it can provide a theoretical basis for wind and sand stabilization.Wind sand liquidity is a complex multi-factor problem and a typical gas-solid two-phase flow in the desert ${ }^{[1]}$. Sand-blocking process is a combination of a single sand macroscopical movement and macrovisual study ${ }^{[2,3]}$. The sand barrier is one of the most effective sand fixation method, the sand barriers is mainly lattice sand barriers, the grass pane sand barries is used most on practical, most studies on sand barriers are also based on lattice sand barriers ${ }^{[4]-8]}$.Some domestic scholars have also conducted some explorations and researches on the shape of sand barriers,such as Qu Jian Jun et al ${ }^{[9]}$ studied the windbreak and sand fixation effects of six types of cotton sand barriers in the three structures of implantable,fenced and bundled structures,various structures and specifications of cotton stalk sand barriers can increase the surface roughness to a certain extent, reduce the near-surface wind speed, slow down the sand movement in the barrier and reduce the amount of sediment transported.Liu Jinhao et al ${ }^{[10]}$ studied the windbreak and sand-fixing capabilities of the regular triangle,square and regular hexagonal grass barriers,the windbreak performance of a square grass sand barrier is obviously smaller than that of a regular triangle and a regular hexagon,the largest surface roughness is a regular triangular grass sand barrier.Wang Chengyu and Wang Yujie et $\mathrm{a}^{[[11]}$ conducted field trials to study the blocking effectiveness of positive semicircular,inverted semicircular and one-shaped sandbag sand barriers,the overall distance and sand erosion of sand barriers,the wind resistance benefits of obtaining a semi-circular sand barrier is best.Hayakawa et $\mathrm{a}^{1[12]}$ studied the flow around the cylinder and the influencing factors of wind speed variation in the flow field. This article proposes a combined ring-shaped sand-barrier,the fluent fluid software and the laminar flow model combined with $\kappa-\varepsilon$ standard model ${ }^{[13]}$ is used to study the sand-resistance characteristics of single and combined ring-shaped sand barriers and their effects on wind speed changes are analyzed, then the structure of the ring-shaped sand barrier is optimized, to get the best structure and arrangement.It can not only solve the problem of high experimental cost and long cycle in the study of windy and irregular regions, and also provide reference for windbreaks and sand fixing problems in areas with irregular wind directions

\section{Sand particles separation principle}

When sand particles move on the aeolian two-phase flow field, it can issubjected of the external forces, the external force including gravity, buoyancy, pressure gradient force, false mass force, basset force, mangus lift force and samffman lift force ${ }^{[14]}$.In this paper, the study of sandblocking characteristic of the ring-shaped sand-barrier is carried out, based on the dual principle of inertial force and centrifugal force (Fig.1).

\subsection{Inertial force principle}

When the sand passes through the ring-shaped sandbarrier windward side, due to due to the principle of the inertial force, a part of the sand particles is blocked when the sand particles knocks against the circular sand-barrier

\footnotetext{
* Corresponding author: liupingyi@cau.edu.cn
} 
windward. If we consider the gravity sedimentation of sand particle obey the law stokes law in aeolian gas-solid two-phase flow, assuming that only gravity and drag act on the sand particle and the particle is in an initial stationary state, then the gravimetric momentum equation and its boundary conditions are when $\mathrm{t}=0$, the sand particle force equation of motion:

$$
\sum F=G_{O}-F_{d}-m \frac{d u}{d t}
$$

In the formula: $\Sigma \mathrm{F}$ is composition of forces $(\mathrm{N}) ; \mathrm{G}_{0}$ is residual gravity (also known as effective gravity), gravity minus the buoyancy $(\mathrm{N}) ; \mathrm{F}_{\mathrm{d}}$ is fluid resistance

$(\mathrm{N}) ; \mathrm{m}$ is the quality of $\operatorname{sand}(\mathrm{kg}) ; \mathrm{u}$ is sand $\operatorname{velocity}(\mathrm{m} / \mathrm{s})$.

Assume that the sand is spherical sand:

$$
\begin{aligned}
& m=\frac{\pi}{6} d_{p}^{3} \rho_{p} \\
& G_{0}=\frac{\pi}{6} d_{p}^{3}\left(\rho_{p}-\rho\right) g \\
& F_{d}=\zeta \frac{\pi d_{p}^{2}}{4} \rho \frac{u^{2}}{2}
\end{aligned}
$$

In the formula: $d_{p}$-grit diameter $(\mathrm{m}) ; \rho_{p}$-grit density $\left(\mathrm{kg} / \mathrm{m}^{3}\right) ; \zeta$-resistance coefficient; $\rho$-gas-solid mixed fluid density $\left(\mathrm{kg} / \mathrm{m}^{3}\right)$.

Will $G_{0}, F_{d}, m$ equivalent to (1) available:

$$
\frac{\mathrm{d}_{\mathrm{u}}}{\mathrm{d}_{\mathrm{t}}}=\mathrm{g} \frac{\left(\rho_{\mathrm{p}}-\rho\right)}{\rho_{\mathrm{p}}}-\zeta \frac{3}{4} \frac{\mathrm{u}^{2}}{d^{p}} \frac{\rho}{\rho_{\mathrm{p}}}
$$

It can be seen from the equation of motion, the acceleration of the sand depends on the residual gravity and air resistance in the static wind. $\mathrm{G}_{0}$ is a constant for a certain size of the sand particle when the sand particle settles in the flowing air, fluid resistance increases with increasing velocity. If the gravity is greater than the buoyancy, the sand particle will accelerate to decline due to its own gravity at the beginning of settlement.

When suspended aeolian around the ring-shaped sand barrier, due to its inertia, the aeolian detaches from the fluid streamline, hits the windward surface of the ringshaped sand barrier, inertial sedimentation occurs on the windward side of the ring-shaped sand barrier (Fig.1). The ring-shaped forms a large vortex, reaches full turbulence in turbulent state, flow rate is very big, the total resistance becomes smaller.

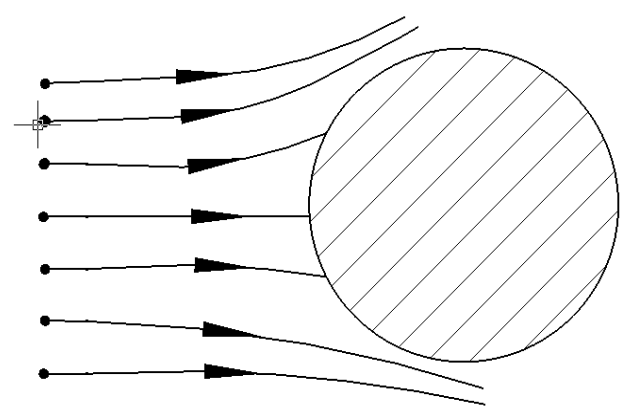

Fig.1. The inertia sedimentation schematicdiagram of sand particles

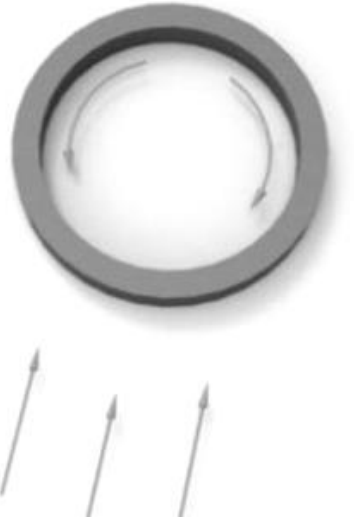

Fig. 2. The centrifugal sand resistance principle of ring-shaped sand-barrie

\subsection{Centrifugal principle}

When the aeolian passes through the ring-shaped sand barrier wall (Fig.2), under the action of centrifugal force, the aeolian rotates movement along the ring-shaped sand barrier wall, by the density differences of sand and fluid, according to the sedimentation coefficient of sand and buoyant density difference to separate sand. During the process of sedimentation, the movement direction and speed of sand particles have the following relations to the density, shape, size and intensity of sand particles.

(1)Centrifugal force of the sand particles is : $F_{C}=\frac{4}{3} \pi r^{3} \rho \omega^{2} R$

$\mathrm{r}$ - Sand particles radius $(\mathrm{m}) ; \rho$ - sand particles density $\left(\mathrm{kg} / \mathrm{m}^{3}\right) ; \omega$-angular velocity $(\mathrm{rad} / \mathrm{s}) ; \mathrm{R}$-airflow rotation radius.

(2)Settlement speed:

When the sand particles is $\frac{4}{3} \pi r^{3} \rho \mathbf{g}$, sand buoyancy is $\frac{4}{3} \pi r^{3} \rho^{\prime} \mathrm{g}$ :

$$
V_{c}=\frac{2}{9} \frac{r^{2}\left(\rho-\rho^{\prime}\right)}{\eta} \omega^{2} R
$$

When the sand particles diameter is $\mathrm{d}$, in the centrifugal field velocity is:

$$
V_{c}=\frac{d^{2}\left(\rho-\rho^{\prime}\right)}{18 \eta} \omega^{2} R
$$

Equation (8) below the unit of gravity, the velocity of the sand particles in the stokes theorem:

$$
V_{c}=\frac{d^{2}\left(\rho-\rho^{\prime}\right)}{18 \eta} g
$$

Above formula: sand sedimentation rate $\left(V_{c}\right)$; the sand particle diameter is medium density $\left(\rho^{\prime}\right)$; gas-solid mixing medium viscosity $(\eta)$.

Sand particles and air is separated under the action of centrifugal force, the greater the centrifugal force of sand, the higher the separation efficiency.

Analytical calculation: When the wind speed is $12 \mathrm{~m} / \mathrm{s}$, $\mathrm{R}=4 \mathrm{~m}, \mathrm{t}=\mathrm{R}-\mathrm{r}=0.1 \mathrm{~m}, \mathrm{~h}=1 \mathrm{~m}$ is the best parameter of sand barrier. 


\section{The simulation of the ring-shaped sand-barrier sand-blocking characteristic}

\subsection{A single ring-shaped sand-barrier}

To model and mesh a single ring-shaped sand barrier by GAMBIT of the fluent software preprocessing software(Fig.3), the size of three-dimensional calculation: the length is $20 \mathrm{~m}$, the width is $10 \mathrm{~m}$, the height is $1.5 \mathrm{~m}$; The diameter of ring-shaped sand-barrier is $8 \mathrm{~m}$,thickness is $0.1 \mathrm{~m}$, height is $1 \mathrm{~m}$;Mesh is divided into $0.2 \mathrm{~mm}$;Boundary conditions: (1) Entrance:speed is $12 \mathrm{~m} / \mathrm{s}$; (2) Export: Free flow; (3) The component of each variable in the normal direction is zero; (4) The remaining boundary conditions using slip-free solid wall boundary conditions ${ }^{[15-16]}$.

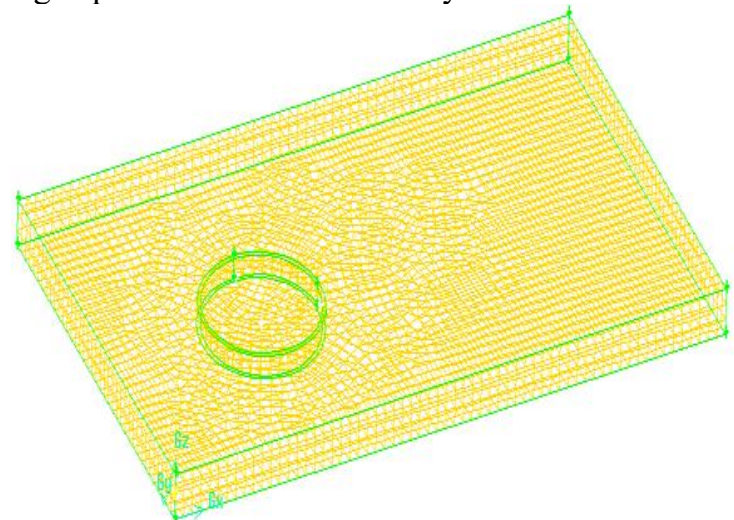

Fig. 3. The grid division graph of a single ring-shaped sandbarrier

The calculation area is modeled by GAMBIT preprocessing software, the model is read into by the FLUENT solver, checking the model file, choosing laminar calculation model, defining fluid physical properties, setting all the parameters, the simulation results are as follows:
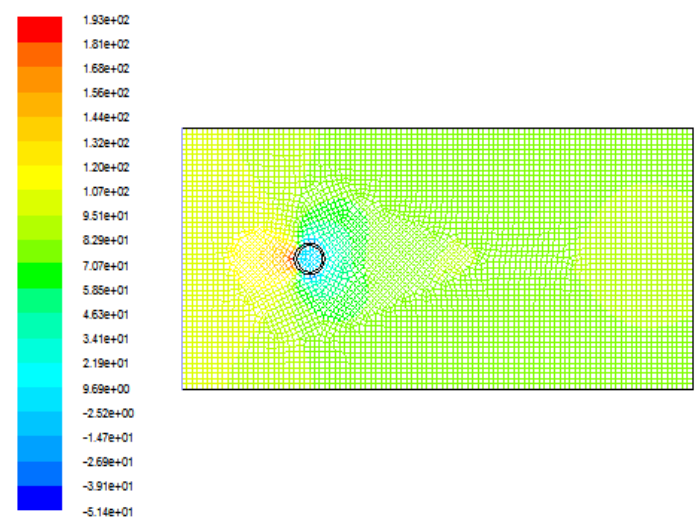

Fig. 4.The pressure nephogram of a single ring-shaped sandbarrier

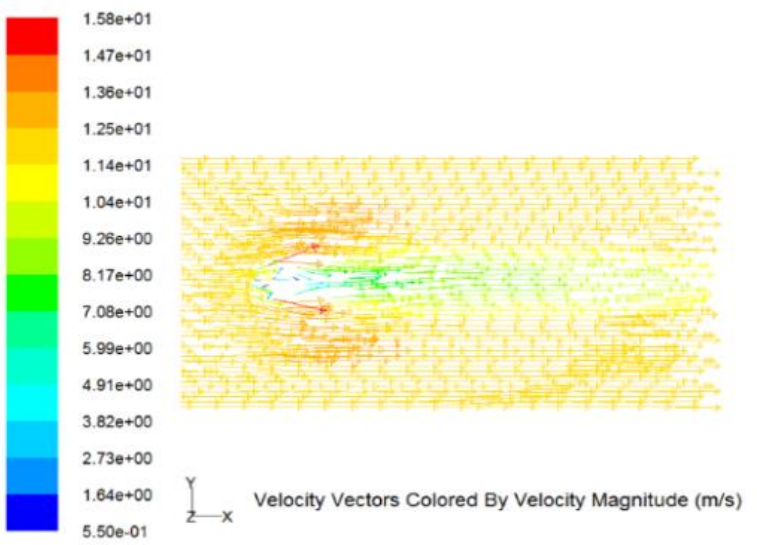

Fig. 5.The velocity vector of a single ring-shaped sand-barrier

In Fig4, the wind pressure is between-5.14e +01 and $1.93 \mathrm{e}+02$, the wind pressure is the largest at the ring sand barrier windward and reduces first and then increase the trend along the radial to the sand barrier leeward in the model area. In Fig5, the speed of the aeolian passing the ring-shaped sand barrier is between $5.71 \mathrm{e}-02$ and $1.77 \mathrm{e}+$ 01 , the speed is the smallest in the ring-shaped sand barrier windward and in the area behind, the wind speed reaches the maximum along on both sides of the tangential direction of the ring-shaped sand barrier in the whole model area, along the radial to the ring-shaped sand barrier leeward,the wind speed increases first and then decreases and the wind pressure is exactly the opposite size. The wind speed gradually increases along the radial direction of the sand barrier surface, until the same with the mainstream wind speed outside, in the sand barrier windward surface the wind speed continues to decline due to sand barrier surface friction, but the main speed of the sand barrier boundary layer is gradually increasing, therefore wind speed will not decline. The prevailing wind speed gradually weakened in the sand barrier leeward, making the wind speed of the ring-shaped sand barrier surface reduced, because of getting the mainstream wind speed outside.

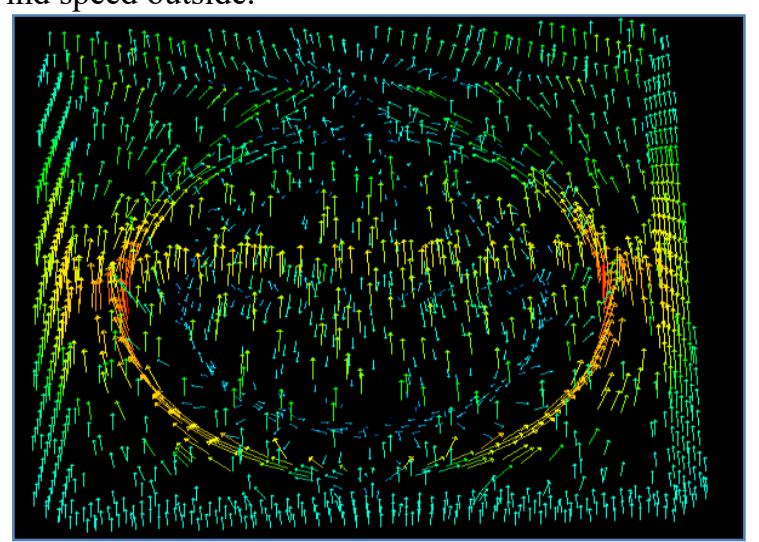

Fig.6. The velocity isocline of a single ring-shaped sand-barrier 


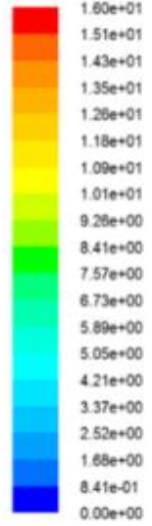

Fig.7.The streamline diagram of a single ring-shaped sandbarrier

It can be seen from Fig 6 that a part of the aeolian has straight the inner wall of the ring-shaped sand-barrier, a part of the aeolian moves along the direction of the center of the wall to move on both sides, due to the inertial force.It can be seen from the fig7 that there are two symmetrical whirling vortices behind the ring-shaped sand barrier, commonly known as Karman vortex,this process is turbulent and it is a steady-state process.

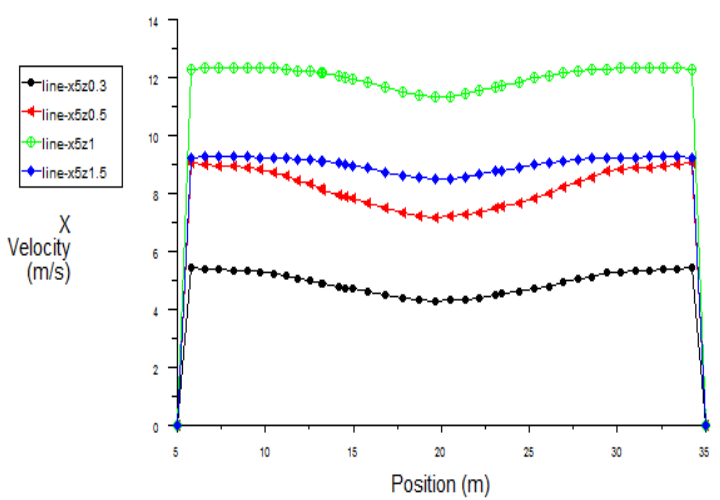

(a) $x=5 m$

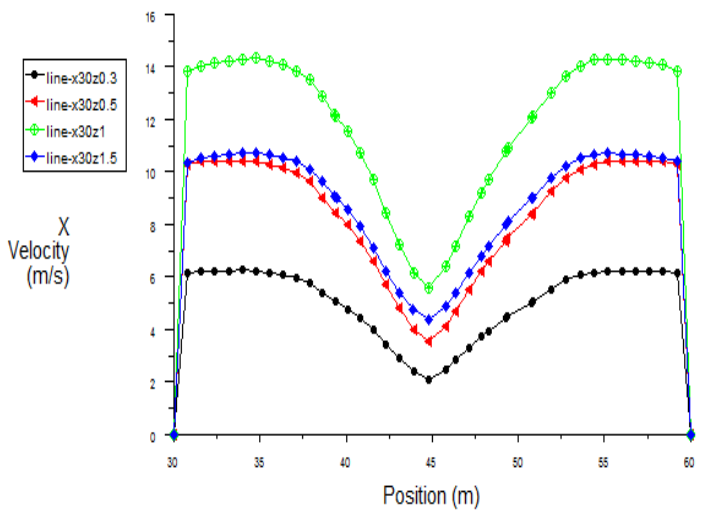

(b) $x=10 \mathrm{~m}$

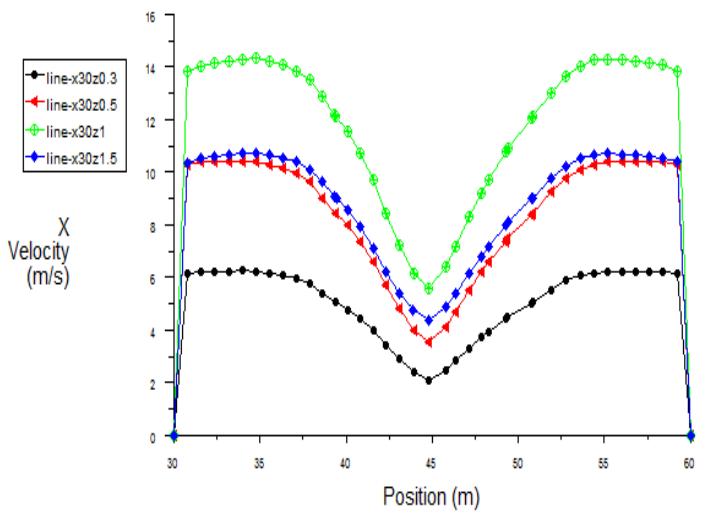

(c) $\mathrm{x}=20 \mathrm{~m}$

Fig. 8. The wind speed before a single ring-shaped sand barrier In Fig8(a), at $5 \mathrm{~m}$ before the single ring-shaped sand barrier, in the area just in front of the sand barrier, at $0.3 \mathrm{~m}, 0.5 \mathrm{~m}, 1 \mathrm{~m}$ and $1.5 \mathrm{~m}$, the change of wind speed is about $1 \mathrm{~m} / \mathrm{s}$, the height has little effect on wind speed changes. The height of the area just behind the sand barrier is $0.3 \mathrm{~m} 、 0.5 \mathrm{~m} 、 1 \mathrm{~m}$ and $1.5 \mathrm{~m}$, the change of wind speed is between $4 \mathrm{~m} / \mathrm{s} \sim 9 \mathrm{~m} / \mathrm{s}$ and $3.5 \mathrm{~m} / \mathrm{s} \sim 6.5 \mathrm{~m} / \mathrm{s}$ respectively, and the wind speed significantly reduces compared to the area before the sand barrier. The wind speed before and after the sand barrier increases with the increase of height, the closer to the ground or closer to the border the smaller the wind speed.

Table 1shows that the mean minimum wind speed at 10 meters after the sand barrier reduces by $49.4 \%$ than at 5 meters before the sand barrier, the average minimum wind speed at 20 meters after the sand barrier reduces to $32.5 \%$ than at 5 meters before the sand barrier.

Table 1 The sand barrier has different distance and high wind speed

\begin{tabular}{cccc}
\hline $\mathbf{Z}$ & $\begin{array}{c}\mathbf{X}=\mathbf{- 5 m} \\
(\mathbf{m} / \mathbf{s})\end{array}$ & $\begin{array}{c}\mathbf{X}=\mathbf{- 1 0 m} \\
(\mathbf{m} / \mathbf{s})\end{array}$ & $\begin{array}{c}\mathbf{X}=\mathbf{- 2 0 m} \\
(\mathbf{m} / \mathbf{s})\end{array}$ \\
\hline $\mathbf{0 . 5 m}$ & 7 & 3.5 & 5.5 \\
$\mathbf{1 m}$ & 11 & 6 & 7.2 \\
$\mathbf{1 . 5 m}$ & 8.5 & 4.2 & 5 \\
$\begin{array}{c}\text { Average } \\
\text { speed( } \mathbf{m} / \mathbf{s})\end{array}$ & 7.7 & 3.9 & 5.2 \\
\hline
\end{tabular}

\subsection{The combined ring-shaped sand barrier}

The combined ring-shaped sand barrier arrangement is the sand barrier staggered arrangement of equal spacing in the desert, the distance between two adjacent sand barriers is greater than the outer diameter of sand barrier,scilice: $\mathrm{d}>$ $2(\mathrm{r}+\mathrm{t})$, three adjacent sand barrier center line was equilateral triangle(Fig.9 ). 


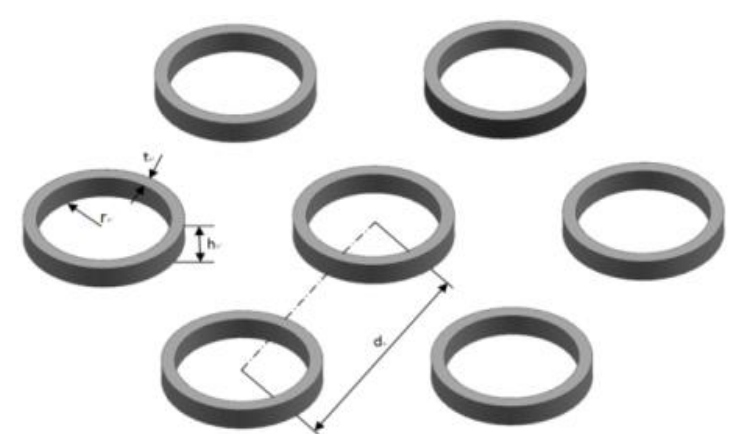

Fig. 9. 3d modeling diagram of combined ring-shaped sand barrier

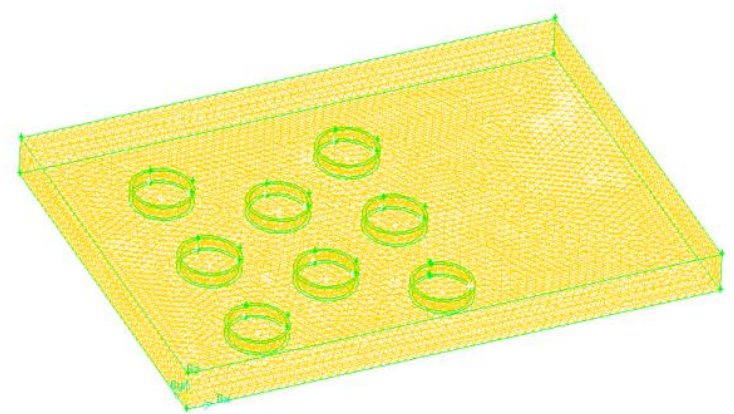

Fig.10 The combined ring-shapedsand barrier grid division map

The same as the study process of sand resistance of a single ring-shaped sand barrier, also use FLUENT software pre-processing software GAMBIT to model and mesh of combined ring-shaped sand barriers (Fig.10), its three-dimensional calculation area model size is: the length is $50 \mathrm{~m}$, the width is $35 \mathrm{~m}$, the height is $3 \mathrm{~m}$, the rest of the conditions are set the same as a single ring-shaped sand barrier, simulation results are as follows.

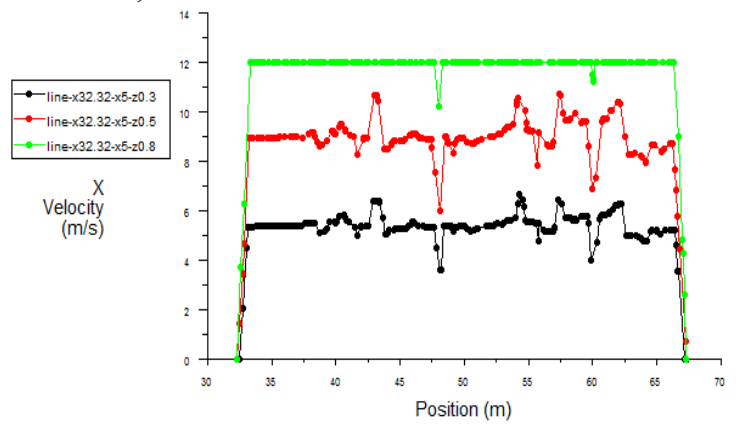

(a) $\mathrm{x}=5 \mathrm{~m}$

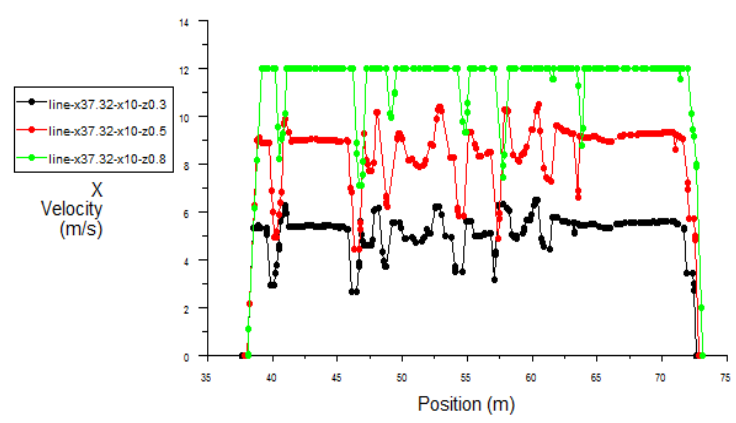

(b) $\mathrm{x}=10 \mathrm{~m}$

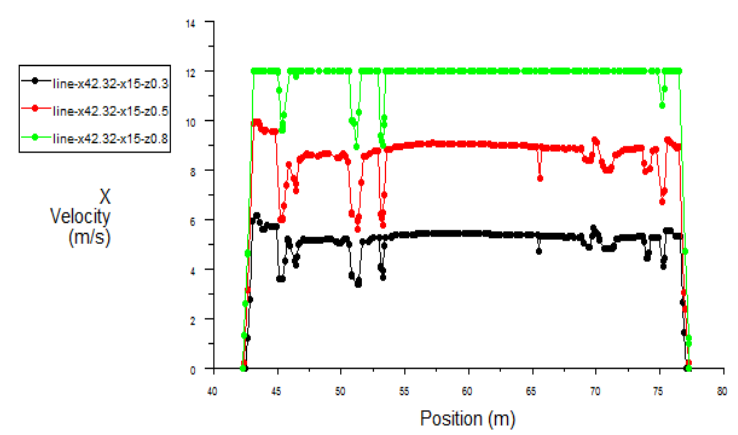

(c) $\mathrm{x}=15 \mathrm{~m}$

Fig.11. Along $x$ direction wind speed after the combined sand -barrier

In Fig.11, the lower the ground clearance the lower the speed and the higher the ground clearance the higher the speed after the sand barrier different distances, eventually approaching the inlet wind speed. At the height of $0.3 \mathrm{~m}$, $0.5 \mathrm{~m}$ and 0.8 when the distance from the sand barrier is $5 \mathrm{~m}$, wind speed is about $5.5 \mathrm{~m} / \mathrm{s}, 9 \mathrm{~m} / \mathrm{s}$ and $12 \mathrm{~m} / \mathrm{s}$, the wind speed are basically the same at $10 \mathrm{~m}$ and $15 \mathrm{~m}$ away from the sand barrier and the wind speed at $5 \mathrm{~m}$, but it has a greater frequency of change fter the sand barrier $10 \mathrm{~m}$, the speed fluctuations become smaller from the sand barrier near or far away, more frequently in the sand barrier interception area.

Table 2 shows, at the same height, after the combining sand barrier at $5 \mathrm{~m}, 10 \mathrm{~m}, 15 \mathrm{~m}$, the average wind speed changes little, but the wind speed is increasing with the increase of the height after the combined sand barriers, with the increase of the distance, the wind speed basically remains unchanged at the same height.Wind speed decreased by $30 \%-58.3 \%$ at different distances and altitude after combined barrier compared with the inlet wind speed.

Table 2. The wind speed of pre and post combined sand barrier at different height and distance

\begin{tabular}{cccc}
\hline $\mathbf{Z}$ & $\begin{array}{c}\mathbf{X}=\mathbf{5 m} \\
(\mathbf{m} / \mathbf{s})\end{array}$ & $\begin{array}{c}\mathbf{X}=\mathbf{- 1 0 m} \\
(\mathbf{m} / \mathbf{s})\end{array}$ & $\begin{array}{c}\mathbf{X}=\mathbf{- 2 0 m} \\
(\mathbf{m} / \mathbf{s})\end{array}$ \\
\hline $\mathbf{0 . 3 m}$ & 5.51 & 5.19 & 5.21 \\
$\mathbf{0 . 5 m}$ & 9.17 & 8.53 & 8.64 \\
$\mathbf{0 . 8 m}$ & 11.97 & 11.51 & 11.83 \\
\hline
\end{tabular}

\section{Conclusion}

(1)Based on the dual principle of inertial force and centrifugal force, analysis and calculation of ring-shaped sand barrier: when wind speed is $12 \mathrm{~m} / \mathrm{s}$, the radius of sand barrier is $4 \mathrm{~m}, \mathrm{t}=\mathrm{R}-\mathrm{r}=0.1 \mathrm{~m}, \mathrm{~h}=1 \mathrm{~m}$, it is the best parameter for a single ring-shaped sand barrier; Ring-shaped sand barrier staggered arrangement of medium-spaced in the desert, adjacent two center distance is greater than the outer diameter of the sand barrier,it is the best layout for way that adjacent three centers from the connection was equilateral triangle.

(2)By using Fluent to simulate the wind speed of sand through the single and combinesand barriers, the average minimum wind speed at 10 meters after a single ringshaped sand barrier decreased $49.4 \%$ compared with 
before the sand barrier, the average minimum wind speed at 20 meters after the sand barrier reduced by $32.5 \%$ compared with that before the sand barrier; Combined sand barrier wind speed at different heights than the inlet wind speed reduced by $30 \%-58.3 \%$. The analysis shows that the average wind speed decreases less than before the sand barrier, the lower the ground clearance, the lower the wind speed, the higher the ground clearance is, the bigger the wind speed is.Compared with the current technology, with more effective anti-desertification effect, it can provide a reference for the actual sand control and sand fixation and be suitable for the desert environment in the complex control of the aeolian control project.

\section{References}

1. Wu sheng-zhi,Ren chun-yong.Numerical simulation of wind-blown sand based on the Eulerian model [J]. Journal of Lanzhou University(Natural Science),2012,48(1): 104-120.

2. S Fohanno,B Oesterle.Analysis of the Effect of Collisions on the Gravitational Motion of Large Particles in a vertical duct[J].International Journal of Multiphase Flow ， 2000,26(2): 267-292

3. Salar Azizi, Dariush Mowla, Goodarz Ahmadi. Numerical evaluation of turbulence models for dense to dilute gas-solid flows in vertical conveyor [J]. Journal of particles, 2012, 553-561.

4. Sun Tao, Liu hu jun,Zhu guo -qing,et al.Timeliness of reducing wind and stabilizing sand functions of three mechanical sand barriers in arid region[J]. Journal of Soil and Water Conservation, 2012,26 (4) :12-16

5. Zhou na, Zhang chun-lai, Tian Jinlu et al. Flow field controlling the concave surface of the semi-buried checkerboards and its characterization by grain sizes of sediments[J]. Research,2014,33(11): 2145-2156

6. Huang qin qin.Research on Numerical Simulation and Feature Structure of Grass Sand Barrier for Mechanised Sand-fixing[D].Beijing forestry university,2014

7. Chen yong.Numerical simulation and optimal design of the vertical array sand trap and checkerboard sandbarrier[D]. Lanzhou University,2014

8. Ma xue-xi,Wang hai-feng,Li sheng yu et al. Comparison of Sand protecting efficiency and Terrain adapted of two checkerboard barriers [J]. Journal of Soil and Water Conservation, 2015,35(2):344-349

9. Ma rui,Wang ji-he, Qu jian jun et al. Study on protective effect of difference types of cotton haulm sand barriers[J]. Journal of Soil and Water Conservation, 2010,24(2):48-51

10. Sun hao,Liu jin-hao,Huang qing-qing et al. Research on the windproof efficiency of polygonal straw sand barrier[J]. Journal of BeijiuR- Forestry University, 2017, 39(10): 90-95
11. Wang chen-feng,Wang Yujie,Yuan li-min et al. Stability and Wind-break Effect of Sandbag Barriers of Different Shapes[J]. Journal of Soil and Water Conservation, 2015,29 (5):7-12

12. Hayakawa M, Hussein A K M F. Turbulence structure in a cylinder wake. Advances in Turbulence[M].Springer-VerlagBerlin Heidelberg, 1987:416-423

13. Yongxiang He, Haibo Zhao, Haoming Wang, et al. Differentially weighted direct simulation Monte Carlo method for particle collision in gas-solid flows[J]. Particuology, 2015(04): 135-145.

14. [14]Zhao Jiang. Numerical Simulation of WindBlown Sand under the Influence of Baffle[D]. Lanzhou University, 2013.

15. Wang fu-jun.Computational fluid dynamics analysis CFD software principles and applications [M]. Beijing: Tsinghua university press,2004

16. Shi F, Huang N. Computational simulation of blow sand fluxes over the surfaces of complex micro to pography[J].Environ mental Modeling \& Soft ware, 2010,25: 362-367. 